\title{
Efeitos de seis manipulações experimentais sobre a aprendizagem de uma nova resposta de fuga
}

\author{
Renato Bortoloti \\ Universidade Federal do Pará \\ Maria de Jesus Dutra dos Reis \\ Júlio C. de Rose \\ Universidade Federal de São Carlos
}

\begin{abstract}
RESUMO
Denomina-se desamparo aprendido o efeito deletério da exposição prévia a situações aversivas incontroláveis sobre a aquisição de novas respostas. Este estudo investigou o efeito de seis manipulações experimentais sobre a aquisição de uma resposta de fuga. Quarenta e oito ratos foram distribuídos em seis grupos de oito. Cada sujeito foi submetido a três sessões experimentais. Na primeira sessão, conforme o grupo a que pertencia, ou o animal recebia choques elétricos que podia interromper, ou recebia choques que só podiam ser interrompidos por outro sujeito, ou não recebia tratamento aversivo. Na segunda sessão, metade dos animais recebia choques incontroláveis e a outra metade não recebia estimulação elétrica. Na terceira sessão, todos os sujeitos tinham a possibilidade de desligar os choques. Os sujeitos que puderam interromper a apresentação dos estímulos na primeira sessão, mesmo aqueles que receberam choques incontroláveis na sessão seguinte, aprenderam a resposta de fuga na última sessão, o que não se verificou entre os animais submetidos inicialmente à incontrolabilidade. Estes resultados replicam o que se obtém normalmente em pesquisas que tratam do desamparo aprendido. Porém, o fraco desempenho dos sujeitos que não receberam choques nas duas primeiras sessões coloca em questão a contingência utilizada para testar a aprendizagem.
\end{abstract}

Palavras-chave: desamparo aprendido; respostas de fuga; ratos

\section{ABSTRACT \\ Effects of six experimental manipulations on the learning of a new escape response}

The deleterious effect of previous exposure to uncontrollable aversive events on subsequent learning is called learned helplessness. This study investigated the effect of six experimental manipulations on the acquisition of an escape response. Forty eight rats were divided in six groups of eight. Three experimental sessions were conducted with each rat. In the first session three different conditions were administered, depending on the group: in the first condition, animals received shocks that they could interrupt; in the second condition, animals were yoked to those in the first condition and could not interrupt shocks, which were interrupted by responses of the yoked animal; in the third condition, animals did not receive shocks. In the second session, half of the animals in each condition received uncontrollable shocks and the other half did not receive shocks. The third session tested whether animals could learn a response to escape shocks. Rats that could interrupt shocks in the first session, even if they received uncontrollable shocks in the second, learned to escape shocks in the test. Those that initially received uncontrollable shocks did not learn to escape in the test. These results replicate earlier findings on learned helplessness. However, the poor performance in the test by the group that did not received shocks leads to questions about the contingency used to evaluate learning.

Keywords: learned helplessness; escape responses; rats

Há evidências experimentais de que organismos expostos a eventos aversivos incontroláveis apresentam uma dificuldade subseqüente para aprender novas respostas operantes, particularmente aquelas negativamente reforçadas (respostas de fuga e/ou esquiva).
Esta interferência da estimulação aversiva incontrolável sobre a aprendizagem posterior tem sido chamada de "desamparo aprendido", denominação que traz consigo uma interpretação do fenômeno que pretende descrever. A despeito de controvérsias que possam ter 
surgido em torno do nome "desamparo aprendido" e da hipótese explicativa por trás dele, o efeito comportamental que ele representa é consistente e teve a sua generalidade verificada em diversas espécies.

O estudo clássico que serviu de base para a primeira descrição desse fenômeno foi constituído por duas sessões experimentais, denominadas de tratamento e teste. Na sessão de tratamento, grupos de dois cães eram presos a arreios que liberavam choques elétricos simultaneamente e com a mesma intensidade. Apenas um dos cães de cada grupo tinha a oportunidade de desligar os choques pressionando um painel com o seu focinho. Se o painel fosse pressionado, o choque cessava também para o segundo animal, cujas respostas não tinham consequiências programadas. Portanto, na sessão de tratamento, os dois cães recebiam choques em quantidades e intensidades iguais, sendo a única diferença crítica entre eles o fato de somente um sujeito em cada grupo ter algum controle exercível sobre esses estímulos. Na sessão de teste, realizada 24 horas depois, os dois cães podiam desligar os choques correndo para o lado oposto da caixa onde se encontravam. Também foi submetido a essa condição um grupo de animais ingênuos, que não tinham recebido qualquer tratamento anterior com choques. Observouse que tanto os sujeitos ingênuos quanto os que receberam tratamento com choques controláveis aprenderam a resposta que desligava os choques, enquanto os cães tratados previamente com choques incontroláveis não aprenderam efetivamente essa resposta de fuga (Seligman, 1975; Seligman \& Maier, 1967). As inúmeras replicações sistemáticas que esse experimento inicial motivou fortaleceram a suposição de que a incontrolabilidade seria uma variável crítica na determinação dessa interferência danosa sobre a aprendizagem futura.

A correlação entre situações aversivas incontroláveis e déficits de aprendizagem tem sido constatada em diversas espécies dentre todos os filos do reino animal, o que confere ao desamparo aprendido uma grande generalidade (Hunziker, 1997). A hipótese explicativa para esse efeito comportamental, conhecida como hipótese do desamparo aprendido, é a de que organismos expostos a situações de incontrolabilidade aprenderiam que suas respostas não produzem conseqüências e deixariam de agir (Peterson, Maier \& Seligman, 1993; Seligman, 1975). Esse aprendizado se daria em função de o indivíduo desamparado ter desenvolvido uma "expectativa" de que o seu comportamento não tem efeito sobre o ambiente e essa "expectativa" o levaria a desenvolver déficits motiva- cionais, cognitivos e emocionais (Maier \& Seligman, 1976; Seligman, 1975). Essa interpretação clássica é, contudo, criticável porque a inferência de que o sujeito desenvolve tal expectativa decorre da análise dos mesmos déficits de aprendizagem que ela se propõe explicar. Trata-se, portanto, de uma explicação circular, problemática do ponto de vista científico.

Uma análise funcional que torna dispensável o recurso a qualquer processo inferido para explicar o fenômeno do desamparo aprendido foi apresentada por Hunziker (2003). Segundo a autora, situações incontroláveis seriam situações em que não existe o reforçamento diferencial de nenhuma resposta, ou seja, nenhuma relação de dependência entre respostas e consequiências pode ser selecionada. Com isso, por exemplo, sob um arranjo experimental com choques incontroláveis,

(...) a alta movimentação corporal eliciada pelos primeiros choques vai ficando apenas sob o controle do processo de habituação, promovido pela apresentação repetida dos choques. Conseqüentemente, a freqüência e intensidade da movimentação corporal caem ao longo da sessão, deixando o sujeito com aparência de 'passivo'. Uma vez que o processo de generalização é parte de toda aprendizagem, e que o teste tem muitos estímulos comuns à fase de tratamento, é provável que o sujeito se comporte, no início do teste, da mesma forma que vinha se comportando na fase anterior. Assim, ele vai se movimentar pouco, o que diminui a chance da resposta selecionada para reforçamento ser emitida. Porém, mesmo que o sujeito emita essa resposta e experimente o reforçamento, a aprendizagem não será facilmente estabelecida, pois envolve uma relação de dependência entre suas respostas e o término do choque que é incompatível com a relação de independência aprendida anteriormente. Sendo opostas essas aprendizagens, é de se esperar que a primeira dificulte a seguinte, produzindo o desamparo (pp. 18-19).

Essa releitura para a hipótese do desamparo aprendido permite prever a baixa atividade geral que se observa no sujeito "desamparado" sem, contudo, recorrer a processos que não se sustentam diante de uma análise lógica.

Nos trabalhos experimentais que abordam o fenômeno do desamparo aprendido, a sessão de teste é normalmente estruturada para que o sujeito tenha a oportunidade de interromper ou evitar a apresentação de um estímulo aversivo ao longo de uma série de tentativas. Para tanto, ele deve emitir uma resposta de fuga/esquiva repetidas vezes na sessão, a cada nova tentativa. Nas condições em que, por exemplo, são 
administrados choques elétricos, cada choque costuma definir uma tentativa e o tempo que o sujeito leva para desligá-lo é habitualmente chamado de latência para a emissão da resposta de fuga na tentativa. Se a resposta não é emitida, o choque é interrompido automaticamente (ou pelo experimentador) depois de um intervalo determinado. A aprendizagem da resposta de fuga é demonstrada pela diminuição consistente da latência para emiti-la ao longo da sessão. Assim, os sujeitos submetidos apenas a tratamentos com eventos aversivos incontroláveis não costumam emitir a resposta de fuga na sessão de teste ou a emitem com latências altas e que não declinam com o passar das tentativas. Por outro lado, aqueles que experimentam anteriormente a possibilidade de controle apresentam latências consistentemente declinantes na sessão de teste, desempenho que demonstra aprendizagem efetiva sob reforçamento negativo.

A avaliação da aprendizagem na sessão de teste deveria ocorrer em situações nas quais sujeitos ingênuos demonstrassem com eficiência o desempenho testado. Se o que se pretende avaliar é a interferência de estímulos incontroláveis sobre a aquisição de novos operantes, a sessão deve ser estruturada para que os sujeitos não submetidos previamente aos estímulos administrados na fase de tratamento demonstrem eficazmente a performance requerida. Esse princípio, embora evidente, foi negligenciado em muitos estudos que abordaram o fenômeno do desamparo tendo ratos como sujeitos. Hunziker (2003) discute esse problema apontando experimentos em que as latências apresentadas pelos animais ingênuos, embora menores do que aquelas verificadas entre os sujeitos submetidos somente a choques incontroláveis, ficavam invariáveis durante toda a sessão (Alloy \& Bersh, 1979; Freda \& Klein, 1976, experimento 3; Jackson, Maier \& Rapaport, 1978, experimentos 1A, 1B e 3; Maier \& Testa, 1975; Seligman, Rosellini \& Kozak, 1975, experimento 2) ou tornavam-se progressivamente mais elevadas no decorrer das tentativas (Freda \& Klein, 1976, experimento 1; Jackson e cols., 1978, experimento 1A). A autora sugere que imperfeições em parâmetros de procedimentos comumente utilizados na época em que os estudos citados foram conduzidos levariam, muitas vezes, os sujeitos ingênuos a emitirem as respostas de fuga sem entrar em contato com as contingências operantes em vigor. Hunziker (2003) relata que introduziu um refinamento metodológico suficiente para colocar o desempenho dos animais ingênuos na sessão de teste dentro do padrão esperado. Buscou-se introduzir uma resposta de fuga que não fosse incompatível com aquelas eliciadas pelo choque, mas que também não fosse facilitada por elas. A resposta selecionada para os ratos emitirem na sessão de teste consistia em atravessar por um orifício situado no alto de uma parede que divida a caixa experimental em dois compartimentos, ou, simplesmente, "saltar por uma janela". Pôde-se, então, constata,r com sujeitos ingênuos, latências iniciais altas e, depois, a queda delas em função da aprendizagem sob reforçamento negativo. Esse delineamento deu mais validade à sessão de teste e permitiu comparações mais legítimas entre desempenhos de grupos submetidos a diferentes manipulações.

No presente estudo, cinco manipulações experimentais envolvendo choques elétricos foram conduzidas para avaliar a interferência delas sobre o desempenho de fuga em ratos. Na sessão de teste, a resposta efetiva consistia em saltar por uma janela. Um dos objetivos foi verificar se a experiência prévia com choques controláveis pode "imunizar" o animal contra efeitos deletérios da estimulação elétrica incontrolável. Também foi avaliado o desempenho de sujeitos ingênuos, que só foram submetidos a choques na sessão de teste.

\section{MÉTODO}

\section{Sujeitos}

Foram utilizados 48 ratos Albino wistar. Os animais eram experimentalmente ingênuos, com idades que variavam entre 100 e 120 dias. Durante o experimento, os animais eram mantidos em gaiolas individuais com alimentação e água ad libitum, seguindo o regime adotado no biotério de ratos do Laboratório de Psicologia da Aprendizagem da Universidade Federal de São Carlos. A iluminação no biotério era controlada de modo que o ambiente permanecia claro por 12 horas a cada dia, das 7 h00 às $19 \mathrm{~h} 00$. As sessões experimentais foram conduzidas durante esse período.

\section{Equipamento}

Foram utilizadas duas caixas paralelepipedais, chamadas de shuttlebox, uma delas tendo piso fixo e a outra piso móvel. As caixas mediam, respectivamente, $46 \times 22 \times 26$ e $50 \times 22 \times 28$ (comprimento, largura e altura), suas paredes verticais eram foscas e suas tampas eram feitas de acrílico transparente. Cada caixa era dividida em dois compartimentos de igual tamanho por uma parede fosca que continha uma passagem retangular $(7,5 \mathrm{~cm}$ de altura e $6,0 \mathrm{~cm}$ de largura, aproximadamente) através da qual o sujeito podia se des- 
locar de um compartimento para outro. Essa parede era removível e posicionável de modo que a passagem podia ficar no nível do piso (funcionando como uma "porta" para o compartimento vizinho) ou $8,0 \mathrm{~cm}$ acima dele (funcionando como uma "janela" para o outro compartimento). O piso das duas caixas era feito de barras metálicas cilíndricas, através das quais choques elétricos de $1 \mathrm{~mA}$ eram administrados pela ação de geradores da marca Grason-Stadler que eram ligados a essas barras. O início de cada choque era controlado pelo experimentador; a sua interrupção podia ser acionada pelo sujeito que se encontrava na caixa de piso móvel se ele passasse de um compartimento para o outro, uma vez que essa caixa dispunha de interruptores que promoviam o desligamento dos geradores se a inclinação do piso mudasse. Esse dispositivo de desligamento do choque pela ação do sujeito podia ficar ativo ou não, a critério do experimentador. A caixa de piso fixo não permitia ao sujeito qualquer controle sobre os choques. Ela ficava acoplada à outra caixa de maneira que, através de seu piso, eram liberados choques de mesma intensidade, duração e em mesma quantidade que na caixa de piso móvel.

\section{Procedimento}

Os animais foram divididos em seis grupos de oito sujeitos e cada sujeito foi submetido a três sessões experimentais separadas entre si por intervalos de 24 horas. Cada grupo foi designado por três letras e cada letra faz referência à condição experimental de uma sessão. A Tabela 1 apresenta os nomes dos grupos e sumariza as condições a que os sujeitos foram submetidos nas três sessões.

Tabela 1. Sumário das condições experimentais a que os sujeitos de cada grupo foram submetidos

\begin{tabular}{|c|c|c|c|}
\hline \multirow{3}{*}{$\begin{array}{c}\text { NOME DO } \\
\text { GRUPO }\end{array}$} & \multicolumn{3}{|c|}{ CONDIÇÕES EXPERIMENTAIS DE CADA SESSÃO } \\
\hline & SESSÃO 1: & SESSÃO 2: & SESSÃO 3: \\
\hline & PRÉ-TRATAMENTO & TRATAMENTO & TESTE \\
\hline CIS & $\begin{array}{l}\text { C - resposta de fuga possível: correr para } \\
\text { o outro lado da caixa atravessando uma } \\
\text { porta. }\end{array}$ & $\begin{array}{l}\text { I - sessão com choques incontro- } \\
\text { láveis. }\end{array}$ & \\
\hline AIS & $\begin{array}{l}\text { A - condição acoplada: mesma quantidade } \\
\text { de choques recebida por CIS, porém } \\
\text { choques incontroláveis. }\end{array}$ & $\begin{array}{l}\text { I - sessão com choques incontro- } \\
\text { láveis. }\end{array}$ & \\
\hline CNS & $\begin{array}{l}\text { C - resposta de fuga possível: correr para } \\
\text { o outro lado da caixa atravessando uma } \\
\text { porta. }\end{array}$ & $\mathrm{N}$ - sessão sem choques. & $\begin{array}{l}\text { S - resposta de fuga possível: saltar por } \\
\text { uma ianela e atingir o outro lado da caixa }\end{array}$ \\
\hline ANS & $\begin{array}{l}\text { A - condição acoplada: mesma quantidade } \\
\text { de choques recebida por CNS, porém } \\
\text { choques incontroláveis. }\end{array}$ & N- sessão sem choques. & \\
\hline NIS & $\mathrm{N}$ - sessão sem choques. & $\begin{array}{l}\text { I - sessão com choques incontro- } \\
\text { láveis. }\end{array}$ & \\
\hline NNS & $\mathrm{N}$ - sessão sem choques. & $\mathrm{N}$ - sessão sem choques. & \\
\hline
\end{tabular}

Os sujeitos de quatro grupos receberam 60 choques elétricos de 30 segundos de duração máxima na primeira sessão. Os animais de dois desses grupos podiam desligar os choques se emitissem uma resposta de fuga definida como correr para o outro compartimento da caixa, atravessando uma "porta" instalada em seu centro (condição C - grupos CIS e CNS). A resposta de fuga de cada um desses animais desligava também o choque que estava sendo administrado, na caixa acoplada, em outro sujeito cujo comportamento não tinha consequiências programadas, de modo que para ele os choques eram incontroláveis (condição A grupos AIS e ANS). Com esse procedimento, a estimulação elétrica administrada nos ratos dos grupos acoplados tinha a mesma frequiência e duração dos choques recebidos pelos animais que podiam desligálos. Os sujeitos de dois outros grupos eram mantidos na caixa experimental por 60 minutos sem receber choques (condição N - grupos NIS e NNS). A segunda sessão acontecia 24 horas após a primeira e, nela, três grupos receberam 60 choques incontroláveis de 10 segundos de duração fixa (condição I - grupos CIS, AIS e NIS) e os outros três permaneceram na caixa por 60 minutos sem receber choques (condição $\mathrm{N}$ - grupos CNS, ANS e NNS). A terceira e última sessão, denominada sessão de teste, era conduzida na mesma caixa experimental para todos os sujeitos. Nela, a divisória cuja abertura havia servido como 
"porta" na primeira sessão era posicionada de maneira invertida, de modo que ela agora passava a funcionar como uma "janela" para o outro compartimento. Nessa sessão, todos os sujeitos tinham a oportunidade de interromper os choques se emitissem a resposta de fuga definida como saltar por essa janela e alcançar o outro compartimento (condição $\mathrm{S}$ - grupos CIS, AIS, CNS, ANS, NIS e NNS). Eram administrados 30 choques e, cada um deles, se não fosse desligado pelo sujeito em intervalo menor, era desligado pelo experimentador após 10 segundos.

Todos os choques eram de $1 \mathrm{~mA}$ e eram liberados a intervalos médios de 60 segundos (amplitude de variação entre 10 e 110 segundos). Cada choque definia uma tentativa e o tempo que o sujeito levava para desligá-lo, nas condições em que isso era possível, era registrado como latência para a emissão da resposta de fuga na tentativa. Os tempos médios que cada grupo levou para emitir a resposta de fuga em cada tentativa foram submetidos à Análise de Variância para medidas repetidas (ANOVOA two-way) e diferenças entre pares foram identificadas por meio do teste de Turkey.

\section{RESULTADOS}

Foram comparados os desempenhos dos grupos de sujeitos na sessão de teste, analisando os tempos médios que os sujeitos de cada grupo levaram para emitir a resposta de fuga "saltar por uma janela e atingir o outro lado da caixa". A Figura 1 apresenta, em blocos de cinco tentativas, a latência média de todos os grupos envolvidos neste estudo.

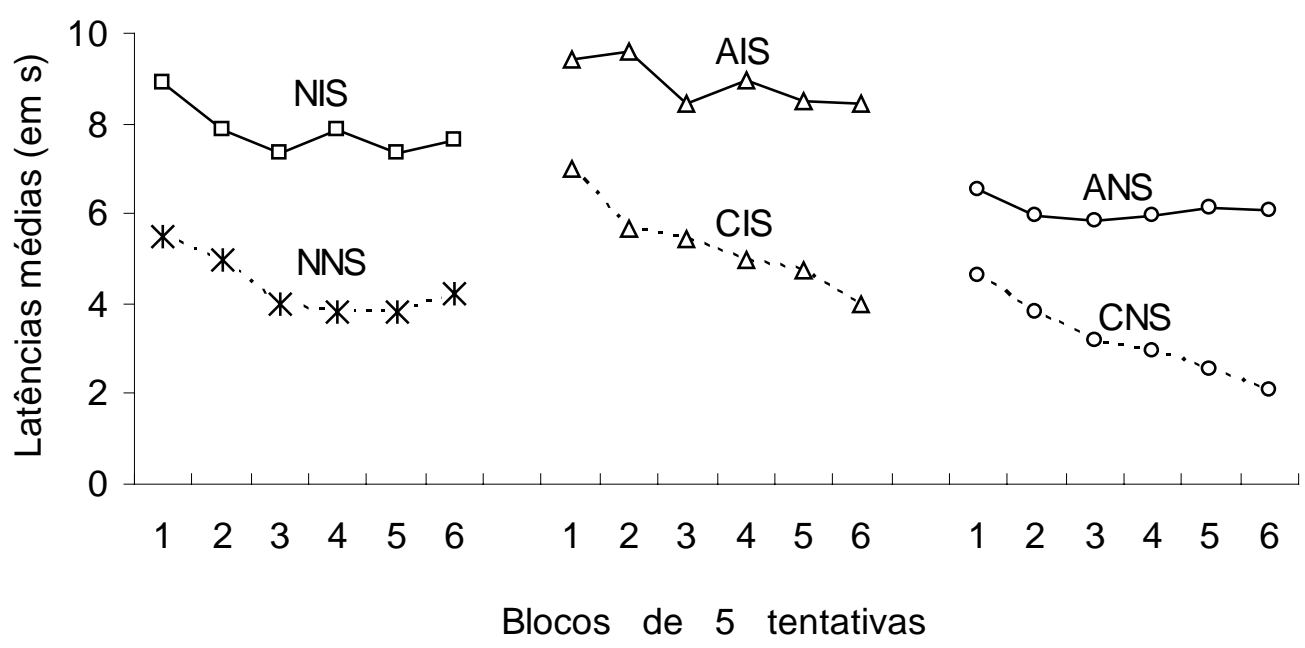

Figura 1. Latências médias para a emissão da resposta de fuga na sessão de teste (saltar por uma janela) apresentadas pelos seis grupos envolvidos neste estudo em blocos de 5 tentativas.

Os grupos que receberam apenas choques incontroláveis (NIS, AIS e ANS) apresentaram as maiores latências para emitir a resposta de fuga na sessão de teste e elas não apresentaram tendência de declínio ao longo das tentativas. Em contraste, os grupos que puderam escapar dos choques na primeira sessão de tratamento (CIS e CNS) apresentaram latências menores e consistentemente declinantes durante a sessão. As latências médias apresentadas pelos sujeitos do Grupo NNS foram menores do que as apresentadas pelos sujeitos dos grupos submetidos apenas a choques incontroláveis $(\mathrm{p}<0,001)$, mas não tiveram declínio consistente ao longo de toda a sessão. A diferença entre os tempos médios para emissão da resposta de fuga entre os grupos acoplados (CIS x AIS e CNS $\mathrm{x}$ ANS) e entre os grupos NNS e NIS foi bastante significativa $(\mathrm{p}<0,001)$.

\section{DISCUSSÃO}

Neste estudo, os sujeitos dos três grupos previamente tratados apenas com choques incontroláveis (NIS, AIS e ANS) tiveram grande dificuldade para aprender ou simplesmente não aprenderam a resposta que interrompia os choques na sessão de teste. Em contraste, os dois grupos que puderam escapar dos choques na primeira sessão de tratamento (CIS e CNS) aprenderam, com eficiência, a nova resposta de 
fuga. É possível interpretar esses resultados à luz da hipótese do desamparo aprendido, mas a comparação entre os grupos perde um pouco de legitimidade quando são considerados os desempenhos dos animais ingênuos (NNS). Embora as latências tenham sido menores do que as apresentadas pelos sujeitos que só receberam choques incontroláveis, o desempenho de NNS ficou aquém daqueles exibidos pelos grupos que experimentaram o controle sobre os choques na primeira sessão. Além disso, o tempo médio gasto pelos sujeitos NNS para emitir a resposta de fuga teve uma tendência de queda durante as primeiras tentativas, mas ela não se manteve. Considerando que o padrão de aprendizagem na sessão de teste deveria ser fornecido pelo desempenho desses animais, os dados obtidos com o Grupo NNS põem em questão a contingência utilizada para testar a capacidade de aprender novas respostas. Se a experiência prévia com eventos aversivos incontroláveis tem efeito deletério sobre a aprendizagem, a avaliação desse efeito deve ocorrer por meio de procedimentos que criem condições nas quais a eficiência de animais ingênuos fique demonstrada de forma inequívoca. Portanto, se o desempenho de fuga apresentado pelos sujeitos NNS não ganhou eficiência ao longo da sessão, a resposta exigida, "saltar por uma janela", não foi plenamente satisfatória para os objetivos deste trabalho.

O fato de haver diferenças estatísticas mostrando que NNS teve desempenho melhor do que os grupos submetidos apenas a tratamentos incontroláveis prova a interferência danosa dos choques incontroláveis. Porém, o desempenho inferior em relação aos grupos CNS e CIS não é suficiente para se concluir que o sujeito fica mais apto a aprender novas respostas quando é exposto a eventos aversivos controláveis do que quando não sofre qualquer experiência traumática. Outras conjunturas merecem ser consideradas nessa análise. No delineamento experimental usado neste estudo, a resposta de fuga efetiva na fase de tratamento era diferente e menos complexa do que aquela que desligava os choques na sessão de teste. Contudo, havia nelas elementos comuns: ambas consistiam em passar por um orifício para atingir o outro compartimento da caixa experimental e implicavam em alta atividade motora. Assim, provavelmente, aprender que atravessar por uma porta podia desligar os choques deve ter facilitado, pelo processo de generalização, a aprendizagem da resposta de saltar por uma janela. Essa talvez seja uma explicação possível para o fato de que os animais com melhor desempenho tenham sido os do Grupo CNS.
Apesar de o delineamento da sessão de teste não ter sido plenamente satisfatório, é interessantes notar o desempenho do Grupo CIS. Um dos propósitos das manipulações experimentais conduzidas foi verificar, através da exposição prévia dos sujeitos a eventos aversivos controláveis, a ocorrência de um efeito "imunizador" contra conseqüências da estimulação aversiva incontrolável. Esse efeito é conhecido e já foi demonstrado em outros estudos (e.g. Alloy \& Bersh, 1979; Anisman, Irwin, Beauchamp \& Zacharko, 1983; Brown, Howe \& Jones, 1990; Hannum, Rosellini \& Seligman, 1976; Ramirez, Maldonado \& Martos, 1992; Yano, 1996; Yano \& Hunziker, 2000). A "imunização" ocorreria porque experiência prévia de que responder produz conseqüências contingentes selecionaria uma relação de dependência entre respostas e conseqüências que poderia prevenir os efeitos deletérios da incontrolabilidade sobre a aprendizagem subseqüente. Neste estudo, os grupos CIS e AIS foram acoplados e receberam as mesmas quantidades de choques durante toda a fase de tratamento. Na primeira sessão, os sujeitos CIS eram os únicos que podiam interromper a apresentação desses estímulos, que eram incontroláveis para ambos os grupos na segunda sessão. Na sessão de teste, os sujeitos CIS apresentaram o desempenho esperado daqueles que aprendem eficientemente uma resposta de fuga e devem emiti-la repetidas vezes: eles saltavam pela janela depois que o choque começava em intervalos cada vez mais curtos durante toda a sessão. Pode-se dizer que eles tiraram proveito da contingência em vigor, ficando muito menos expostos à estimulação aversiva do que os sujeitos do grupo acoplado. Mesmo a exposição a sessenta choques incontroláveis, cada qual com dez segundos de duração fixa, não foi suficiente para suprimir dos sujeitos CIS a capacidade de responder diante de uma nova situação.

O fenômeno do desamparo aprendido foi sugerido como um modelo experimental para a depressão e tem dado valorosas contribuições para o estudo dessa patologia. Defensores do modelo sugerem que a depressão humana é causada pela perda de controle que é experimentada em função de eventos traumáticos. Isso é defendido por um grande número de paralelos estabelecidos entre a depressão e o comportamento "desamparado". São vistas similaridades quanto à perda de peso, letargia e falta de motivação. Além disso, há também paralelos estabelecidos entre os mecanismos bioquímicos e o tratamento dos dois fenômenos. Antidepressivos, inibidores da MAO etc., que são efetivos no tratamento da depressão também podem prevenir e reverter o desamparo aprendido. Ansiolíticos e neu- 
rolépticos, inefetivos no tratamento da depressão, também não funcionam para prevenir ou reverter o desamparo (Eisenstein \& Carlson, 1997; Sagen, Sortwell \& Pappas, 1990). Resultados obtidos neste estudo (e em muitos outros) parecem indicar que o desamparo aprendido não se manifestaria se o organismo tivesse a chance de um contato prévio com uma contingência em que as suas respostas sejam efetivas, ou seja, o desamparo pode ser prevenido comportamentalmente. Criar contingências adequadas para que as pessoas possam experimentar consequiências reforçadoras para as suas ações deveria envolver o esforço de todos que, no mínimo, desejam ver diminuída a incidência epidêmica dos casos de depressão em nossa sociedade.

\section{REFERÊNCIAS}

Alloy, L. B. \& Bersh, P. J. (1979). Partial control and learned helplessness in rats: control over shock intensity prevents interference with subsequent escape. Animal Learning \& Behavior, 7, 157-164.

Anisman, H., Irwin, J., Beauchamp, C. \& Zacharko, R. (1983). Cross-stressor immunization against the behavioral deficits introduced by incontrollable shock. Behavioral Neuroscience, $97,452-461$

Brown, G. E., Howe, A. R. \& Jones, T. E. (1990). Immunization against learned helplessness in the cockroach (Periplaneta americana). Psychological Reports, 67, 635-640.

Eisenstein, E. M. \& Carlson, A. D. (1997). A comparative approach to the behavior called 'learned helplessness'. Behavioural Brain Research, 86, 149-160.

Freda, J. S. \& Klein, S. B. (1976). Generality of failure-to-escape (helplessness) phenomenon in rats. Animal Learning and Behavior, 4, 410-40.

Hannum, R. D., Rosellini, R. A. \& Seligman, M. E. P. (1976). Learned helplessness in the rat: retention and immunization. Developmental Psychology, 12 (5), 449-454.

Hunziker, M. H. L. (1997). Um olhar crítico sobre o estudo do desamparo aprendido. Estudos de Psicologia, 14 (3), 17-26.
Hunziker, M. H. L. (2003). Desamparo aprendido. Tese de Livre Docência apresentada ao Instituto de Psicologia da Universidade de São Paulo. São Paulo, SP.

Jackson, R. L., Maier, S. F. \& Rapaport, P. M. (1978). Exposure to inescapable shock produces both activity and associative deficits in the rat. Learning and Motivation, 9, 69-98.

Maier, S. F. \& Seligman, M. E. P. (1976). Learned helplessness: theory and evidence. Journal of Experimental Psychology: General, 105, 3-46.

Maier, S. F. \& Testa, T. J. (1975). Failure to learn to escape by rats previously exposed to inescapable shock is partly produced by associative interference. Journal of Comparative and Physiological Psychology, 88, 554-564.

Peterson, C., Maier, S. F. \& Seligman, M. E. P. (1993). Learned helplessness: a theory for the age of personal control. New York: Oxford University Press.

Ramíres, E., Maldonado, A. \& Martos, R. (1992). Attributions modulate immunization against learned helplessness in humans. Journal of Personality and Social Psychology, 62 (1), 139-146.

Sagen, J., Sortwell, C. E. \& Pappas, G. D. (1990). Monoaminergic neural transplants prevent learned helplessness in a rat depression model. Biological Psychiatry, 28, 1037-1048.

Seligman, M. E. P. (1975). Helplessness: on depression, development and death. San Francisco: W. H. Freeman.

Seligman, M. E. P. \& Maier, S. F. (1967). Failure to escape traumatic shock. Journal of Experimental Psychology, 74 (1), 1-8.

Seligman, M. E. P., Rosellini, R. A. \& Kozak, M. J. (1975). Learned helplessness in the rat: time course, immunization and reversibility. Journal of Comparative and Physiological Psychology, 88, 542-547.

Yano, Y. (1996). Desamparo aprendido e "imunização" com diferentes respostas no treino e teste. Dissertação de Mestrado apresentada ao Instituto de Psicologia da Universidade de São Paulo. São Paulo, SP.

Yano, Y. \& Hunziker, M. H. L. (2000). Desamparo aprendido e imunização com diferentes respostas de fuga. Acta Comportamentalia, 8, 143-166.

Recebido: 09/05/2006

Revisado: 04/09/2006

Aceito: 02/11/2006

\section{Sobre os autores:}

Renato Bortoloti: Psicólogo, mestre em Psicologia: Teoria e Pesquisa do Comportamento pela Universidade Federal do Pará e doutorando pelo Programa de Pós-graduação em Teoria e Pesquisa do Comportamento da mesma universidade.

Maria de Jesus Dutra dos Reis: Doutora em Psicologia pela Universidade de São Paulo e Professora Adjunta do Departamento de Psicologia da Universidade Federal de São Carlos.

Júlio C. de Rose: Doutor em Psicologia pela Universidade de São Paulo e Professor Titular do Departamento de Psicologia da Universidade Federal de São Carlos.

Endereço para correspondência: Renato Bortoloti - Programa de Pós-Graduação em Teoria e Pesquisa do Comportamento - Centro de Filosofia e Ciências Humanas - Universidade Federal do Pará - Campus Universitário do Guamá - 66075-110 Belém - PA - Endereço eletrônico: r.bortoloti@terra.com.br. 